\title{
Versatile Intelligent Portable Robot Control Platform Based on Cyber Physical Systems Principles*
}

\author{
Victor VLADAREANU ${ }^{1}$, Ioan DUMITRACHE ${ }^{2}$, Luige VLADAREANU ${ }^{1, *}$, Ioan Stefan SACALA ${ }^{2}$, \\ Gabriela TONT, ${ }^{3}$, Mihnea Alexandru MOISESCU ${ }^{2}$ \\ ${ }^{1}$ Institute of Solid Mechanics of the Romanian Academy, \\ 15, C-tin Mille Street, Bucharest, 010141, Romania, \\ vladareanuv@gmail.com; luigiv@arexim.ro \\ * Corresponding author \\ ${ }^{2}$ University "Politehnica" of Bucharest, \\ 313, Splaiul Independentei, Bucharest, Romania \\ ioan.sacala@acse.pub.ro \\ ${ }^{3}$ University of Oradea, \\ 1, University Street, Oradea, 410087, Romania \\ gtont@uoradea.ro
}

Abstract: This paper studies humanoid and walking robot hybrid dynamic control through optimization of the intelligent control methods in order to increase the robot stability on uneven ground, at variable robot speed and disturbing loads. The robots performances, controlled by the intelligent control interfaces are analyzed and the virtual projection method is applied to the Versatile Intelligent Portable Robot Platform VIPRO developed as a Cyber Physical Systems. The results lead to higher performance, stability, reliability, robustness and efficiency in approaching the predictable robot motion and in the development of new technological capabilities of the physical systems with applications in the field of robotics.

Keywords: humanoid or walking robots control; cyber physical systems; virtual projection method; stability of walking robots; versatile, intelligent, portable robot control.

\section{Introduction}

Three important industrial revolutions have influenced manufacturing: first, coal, steam and mechanization, second, electricity motors and machines and third Computers, Information Technologies and Internet. The fourth major industrial revolution is currently emerging and is enabled by Future Internet paradigms such as Internet of Things and Internet of Services. Thus, the integration of these emerging technologies in industrial environment is enabled by the Cyber Physical Systems paradigm.

The emerging vision for manufacturing systems is encapsulated in Industrie 4.0 concept developed with the aid of the German government, with the aim of implementing Smart Factories.

\footnotetext{
* This paper is based on a former presentation entitled "Haptic Interfaces for Compensating Dynamics of Rescue Walking Robot" made at the International Conference on Communication, Management and Information Technology 2015. In the current paper we updated the model in order to include the Cyber-Physical System for future integration in the context of Internet of Things. Also, we defined several methods for CPS and optimized the intelligent control.
}

Industrie 4.0 vision integrates Cyber-Physical Systems including sensors and actuator networks, intelligent network control systems and human in the loop principles.

In this paper, according to similar researches in the field of humanoid or walking robots real time control [1-4], in order to increase mobility and stability of walking robots movement, the authors' main challenge is to develop a federated, adaptive, scalable, interoperabilityfocused robotic system in the field of CyberPhysical Systems.

\section{Robotic Systems as Cyber Physical Systems}

In order to implement an intelligent control platform within a Cyber-Physical System, the following modeling aspects must be applied: Networked Control System modeling, Intelligent Informational System, Sensor modeling, interoperability between P2P entities and Machine 2 Machine (M2M) communication. Also, the intelligent control platform will act both as service provider and service consumer. 
Communication channels within the platform are represented as a link between the data acquired from the sensors, the intelligent decision support systems and the control system. The environmental channel allows the robot to identify the objects from the environment as well as user's commands.

Social Awareness is a component of the robot that follows a behavior composed of socially acceptable conditions and provides social cues indicating the way the robot tries to maintain engagement. Situation Awareness (SA) represents the way the platform understands the environment. Three levels of SA have been explored: perception, comprehension and projection.

Challenge: development of federated, adaptive, scalable, open-architecture, interoperabilityfocused robotic systems.

Principles for Design of CPS-Robotics Systems

- Sensing and Actuating

- sensor and actuator networks technologies

- Semantic enabled sensors

- service oriented architecture

- data modeling, semantics, ontologies

- real-time data management,

- Human in the loop

- Human to Machine - > human - robot interaction

- Machine to Human -> robot - human interaction

- human-machine interface, augmented and virtual reality

- context awareness multiple robot management, collaboration,

- Robot control

- Network control systems

- Robot collaboration

- service oriented robotic systems

- intelligent decision support systems integrated into robotic systems

- Machine to Machine collaboration - > Machine to Machine learning

- semantic technologies

Principle 1: CPS modelling methods:

- System modeling: SystemML

- Networked Control System modeling
- traceability within Intelligent Informational System

- Sensor modeling: SensorML

- Machine 2 Machine communication

- interoperability between P2P entities

- $\quad$ M2M learning

ROBOTIC SYSTEM as a Complex, Adaptive, Networked System, integrating various entities (objects): robots, smart devices, humans.

Principle 2: The mobile robot acting as both service provider and service consumer.

- message routing

- REST service

- semantic enabled data acquisition

- context awareness

- Future internet as supporting environment

- Interoperability-focused (data/processes/knowledge)

Principle 3: Human in the loop

- Communication channels expressed as a link between the data acquired from the sensors, the intelligent decision support systems and the control system.

- Environmental Channel that allow the robot to identify objects as well as user's actions/commands.

- Social Awareness as an ability of the robot to follow a behavior that respects socially acceptable conditions and gives readable social cues indicating how the robot tries to maintain engagement during the work scenario.

- Situation Awareness (SA) as described by "knowing what is going on around you". Three levels of SA have been explored: perception, comprehension and projection.

Cyber-Physical Systems defined by the National Science Foundation: "engineered systems that are built from, and depend upon, the seamless integration of computational algorithms and physical components"

CPS research is directed at achieving the following:

- "capability, adaptability, scalability, resiliency, safety, security, and usability 
that will far exceed the simple * embedded systems of today"

- extending control theory in order to handle

- networks of devices

- Networked control systems

- large-scale integration of the physical and cyber worlds

- $\quad$ systems of systems

\section{Robot Control Strategy applied to Cyber Physical Systems}

The robot control strategy needs to take into account the various known issues involved in moving walking robots, while incorporating new capabilities for a wide array of circumstances. It is therefore necessary to adopt new high level intelligent algorithms for undertaking actions such as walking up or down slopes, overcoming or avoiding obstacles and handling unknown rough terrain [5-7].

The walking robot is considered as a set of articulated rigid bodies, which are standing as a platform and leg elements. The static stability problem is solved by calculating the extremity of each leg position according to the system of axes attached to the platform, with the origin at its center of gravity. The process of walking and stepping on a rough surface for an extended period is particularly difficult to predict and plan for. This instability can eventually lead to the overthrow of the robot. It is thus necessary to develop high-level intelligent algorithms $[3,5,11]$ when attempting to introduce new capabilities for walking robots.

The Cyber-Physical Systems (CPS) concept can be defined of the following main elements: "Cyber", which refers to computation, communication, and control that is discrete, logical and "Physical" which relates to natural and human-made systems governed by the laws of physics and operating in continuous time. A generic CPS is composed of: physical objects, sensors, actuators, computing devices (e.g. controllers) and communication networks [8-10].

The mathematical modeling of the center of gravity position, allows the control of the walking robots when moving on complicated terrain, if is fulfilled the condition for stability. This leads to the condition that the vertical projection of the center of gravity of the system must be inside the support polygon. As the positions of the centers of gravity for each element of the leg mechanisms in known in relation to their own systems, the relation can be determined for the position coordinates of the robot center of gravity, necessary for the real time control of robot stability:

$$
X_{G}^{k}=\frac{m_{0} \cdot X_{O}^{k}+\sum_{i=1}^{6} \sum_{j=1}^{3} m_{j}^{i} \cdot X^{k} \cdot G_{j}^{i}}{\sum_{i=1}^{6} \sum_{j=1}^{3} m_{j}^{i}}
$$

where $\mathrm{X}^{\mathrm{k}}=\{\mathrm{X}, \mathrm{Y}, \mathrm{Z}\}(\mathrm{k}=1.2 .3),. \mathrm{j}=1-4$ and $\mathrm{i}=1-6$ for a hexapod walking robot and $\mathrm{i}=1-4$ for a four-legged robot. Knowing the center of gravity position, the speed $\dot{X}_{G}^{k}$ and acceleration $\ddot{X}_{G}^{k}$ are determined by differentiation.

Maintaining the vertical of the center of gravity within the support surface is all the more difficult if the robot is moving on a slope. In this case, maintaining stability depends on the transported load $\left(\mathrm{f}_{\mathrm{i}}\right)$ and the distance $\mathrm{X}_{\mathrm{C}}$ from the support point surface to the center of gravity. Stability is obtained by reducing the component $\mathrm{X}_{\mathrm{C}}$ when increasing the load $\left(\mathrm{f}_{\mathrm{i}}\right)$, depending on the movement slope. An innovative control method, applicable to CyberPhysical Systems, which practically eliminates instability and has a fast control loop response is presented $[6,11,13]$. The fuzzy multi-stage control (MS) multiple rule bases where the result of a rule inference is sent to the next level [15]. This way, the most important dimensions of the inference can be grouped into smaller sets and combined with the base rules. The results of the MS rule base structure for position control $\mathrm{P}$ are sent to the rule base inference for force-position control FP. By applying fuzzy logic control there is obtained a smooth transition from position control to force and position control in the case of walking robot movement. As relates to the control of walking robot movement, 7 types of models for dynamic control and two hybrid force-position control loops were established, with the aim of producing online control loops for stable walking $[6,17,20]$. The proposed architecture allows the system to be tested in different conditions, in order to fulfill all the necessary requirements. The system will be easy to be configured in different environments, depending on the circumstances. Two models will be integrated using VIPRO platform, one based on specific open source platforms 
"VIPRO Platform Specific Environment" and the other one focusing on intelligent control methods by integrating functionalities from multiple existing platforms as services or plugins "VIPRO Integration Environment".

The final result is the humanoid or walking robots control algorithm, simulated in real time through virtual experimentation using the virtual projection method, also known as Vladareanu-Munteanu [18], presented in the next chapter.

\section{Building of the VIPRO Cyber Physical Systems}

The open architecture $[13,18]$ of the VIPRO control system [16, 21], is presented below. SCMC is the classic control system, which drives the servo-actuators MS1, $\mathrm{MS}_{\mathrm{m}}$, and receives input signals from $\mathrm{TM}_{1}-\mathrm{TM}_{\mathrm{m}}$ transducers, with $m$ being the robot degrees of freedom. Load actuators are rigidly coupled to the servo-actuator modules receiving control signals from a MCS load controller module.

Walking robots stepping on uneven or unstructured terrain is a difficult process to understand, being a repetitive series of tilting and unstable movement which can result in instability. To this end, ZMP compensation is used in conjunction with inertial information provided by the sensors, to mitigate these effects [5, 13]. This is done by generating compliant walking patterns, compensating for the ZMP in real time in the support phase, leg joint damping control and using the angular speed of the platform for stable position and stepping control.

Virtual projection method applied to VIPRO Cyber Physical Systems can integrate the facilities brought by several existing robot platforms. Our focus is to create a platform integrating the Platform integration module. VIPRO platform offers the possibility of direct control of the robot simulation or via a user interface:

- a way of communication with mobile robots independently or using intelligent interfaces

- a dedicated management module for different existing robotic platforms

- a module that implements intelligent control techniques and one for motion planning

The innovative virtual projection method by Vladareanu-Munteanu allows the introduction of complex control algorithms for dynamic walking robots, virtualization and testing of positioning and joint trajectories, the feed-back forces and walking robots dynamic control.

The advantages of the proposed method consist of real-time robot balance control, gait control and predictable motion control providing increased mobility and stability in order to achieve higher performance.

All these trends, including the evolution of technology and the increasing need for large scale systems, have led to great research interest for integration of the virtual projection method into VIPRO platform using Cyber Physical Systems.

Using ZMP and inertial information [3, 12-14, 16], strategies are developed for dynamic gait

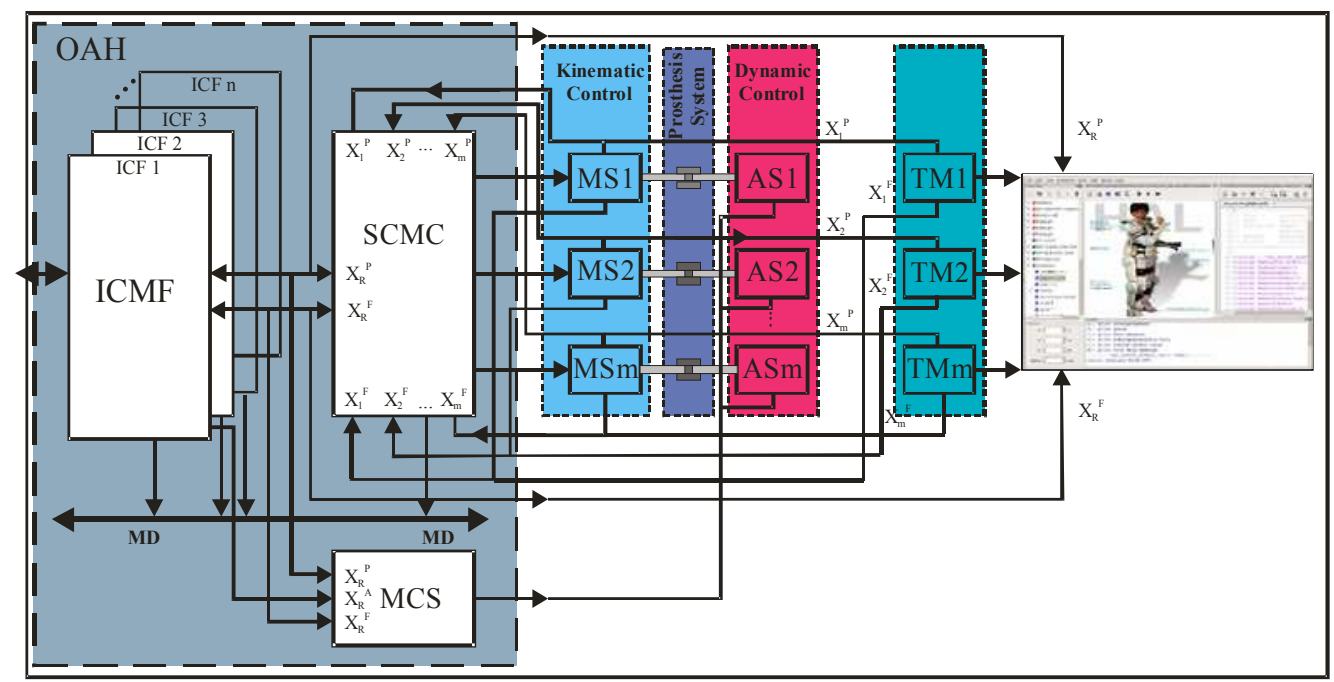

Figure 1. Virtual projection method by Vladareanu-Munteanu applied to VIPRO Cyber Physical Systems 
control. This includes compliant walking pattern generation, real-time ZMP compensation in the support phase, joint damping control and stepping control.

\section{The Optimization of Intelligent Control Methods for Cyber Physical Systems}

Cybernetic physical system allow for synergies of the physical and cyber world. The optimization of Cyber Physical Systems through intelligent control methods can bring about unprecedented applications, allowing the mobile autonomous robot to adapt to uneven ground, through real time control, without losing its stability during walking $[3,16,18]$. Progress in Cyber Physical Systems, however, requires an availability of co-simulation platforms, which can capture both the physical dynamics, as well as the communication component. To this end, the VIPRO platform architecture, presented in Figure 2, in correlation with the virtual projection method (Figure 1) is a comprehensive co-simulation platform for Cyber Physical Systems.

The versatile, intelligent and portable robot platform VIPRO presented in this paper contains three intelligent control interfaces (ICs), for modelling using the human adaptive mechatronics methods of the walking robot. Human adaptive mechatronics $[15,11,22]$ are intelligent electrical-mechanical systems that are able to adapt themselves to the human's skill in various environments and providing assistance in improving the skill, and overall operation of the combined human machine system to achieve the improved performance.

The main modules of the platform's architecture are the intelligence control interface module, which uses advanced control strategies adapted to the robot environment such as extended control - Extenics [18-21], neutrosophic control $[22,23]$, human adaptive mechatronics, implemented through fast processing techniques and real time communication.

The newly developed platform enjoys a number of indispensable characteristics. In the process of developing the steps for platform engineering, there are developed an array of alternative intelligent control methods which could prove beneficial in other future tools that combine different simulator environments.

The optimization of the Cyber Physical Systems is undertaken in this paper mainly through the integration of the interface for intelligent neutrosophic control using the RNC (Robot Neutrosophic Control) method, extended control interface through Extenics method (ICEx) and haptic robot control interface (CRH).

The development strategy for the CPS implies developing the robot virtual 3D environment,

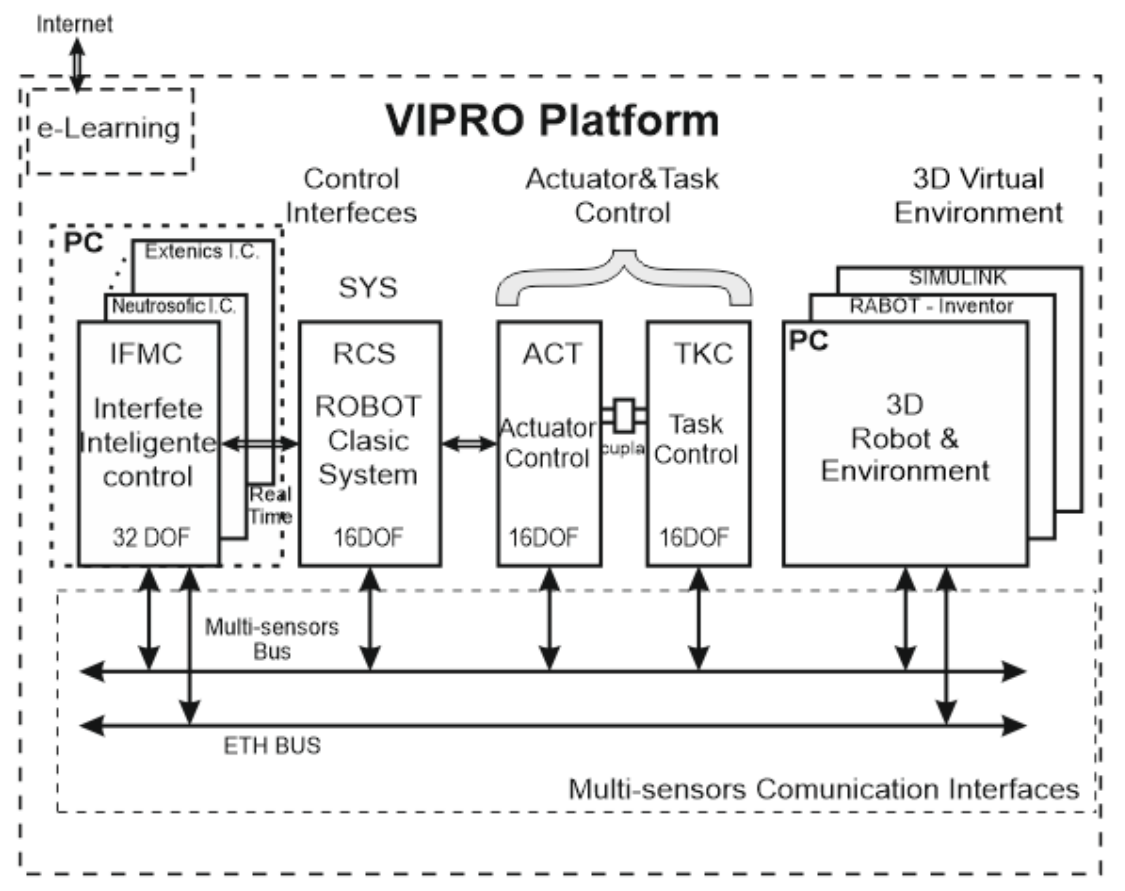

Figure 2. The architecture of the VIPRO platform adapt to Cyber Physical Systems 
through CAD projections of the NAO robot and the RABOT rescue robot. The models are then exported to the VIPRO 3D virtual simulation platform and running real time control simulations in the 3D virtual environment through a powerful robot simulator with practical application in the NAO robots and search \& rescue robots such as RABOT. This is correlated with implementing the multi-sensor communication interface which contains the multi-sensor bus and the Ethernet communication bus, in order to ensure high speed data transfer between platform modules.

Validation of the CPS is done through integration into the microcontroller system of the intelligent control interfaces, after which the improved performance is tested on a versatile intelligent portable robot (VIPRO) platform. The following will present the open architecture system and the intelligent control interfaces which display the capabilities of the VIPRO CPS.

\section{Open Architecture System and Control Interfaces of the VIPRO Cyber Physical Systems}

The Cyber Physical Systems, developed as a future generation of engineering systems, require the close integration of computing, communication and control technologies with the aim of obtaining the stability, performance, reliability, robustness and efficiency in approaching physical systems with applications in the field of robotics [3,11, 24, 25,27].

Although the specific context of the issues and challenges facing CPS today is different from the past, the basic aim of developing control systems through the integration of technologies from computing and communication is rooted in open architecture system development. The concept of distributed robot control assumes an interaction between sensors and peripheral equipment such as the LAN network for offline communication, fast CAN network for real time control, digital and analogue interface modules. By employing an open architecture, the informational flux load on the main controllers is effectively diminished due to a master-slave type control system between the central PLC unit, supplemented by the inputoutput devices and communication lines functioning between the main control system and the PC system.

The Cyber Physical Systems of the versatile, intelligent and portable robot platform VIPRO, presented in Figure 4 was conceived with an open architecture in distributed and decentralized structure in order to allow for easy development of new applications or the addition of hardware or software modules for new control functions. The control system

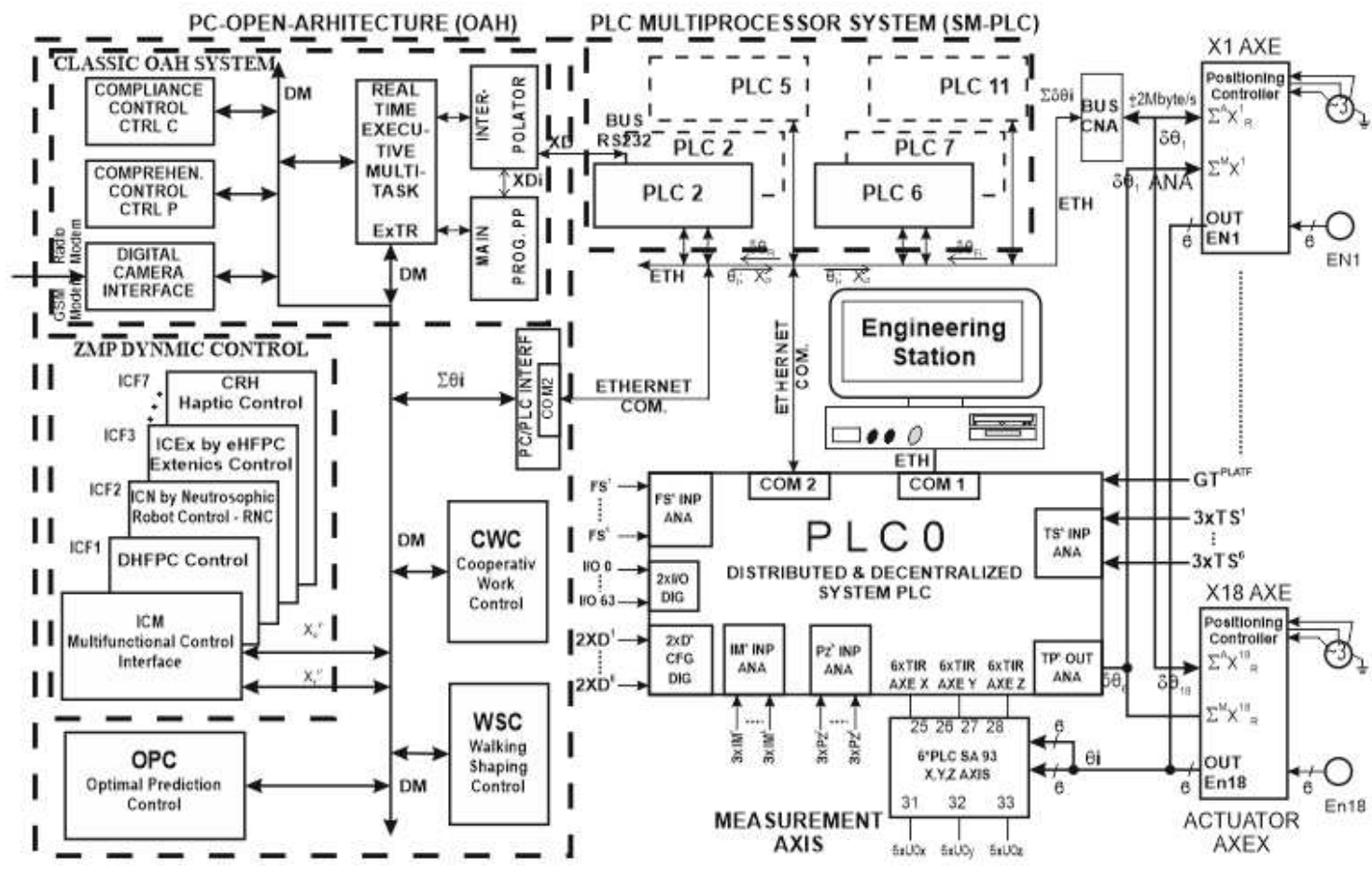

Figure 3. Open Architecture System and intelligent interfaces of the VIPRO Cyber Physical Systems 
integrates the neutrosophic control interface (ICN) by applying the neutrosophic robot control method (RNC), extended control interface (ICEx) by applying extended hybrid force-position control method (eHFPC) and the multifunctional control interface (ICM) which ensures real time synchronisation of the intelligent control interfaces (ICF1-ICF7). In addition, the haptic robot control interface $(\mathrm{CRH})$ is designed for robot navigating on uneven terrain and uncertain environments.

The multifunctional control interface (ICM) with a very important role for the versatility and portability of CPS allows the real time control, priority control and data exchange monitoring between $\mathrm{n}$ function control interface (ICF1 - ICF7) and the classical mechatronic control system of the robot $[3,18]$, composed of the distributed and decentralized system $\mathrm{PLC}_{0}$, positioning controllers on each robot DOF, engineering station and the PLC multiprocessor system SM-PLC. The function control interfaces are connected through a high speed data bus (MD).

The basic elements of a standard structure for Cyber Physical Systems of the versatile, intelligent and portable robot platform VIPRO using the PC-OPEN Architecture OAH are presented below. By using the PLC0 communication function block, supported by a RS232 serial interface, the values of the current absolute angular displacements $\Sigma \theta$ ci are sent from the PLC to the PC. The PC sends to the PLC continuously the reference position for each axis $X_{D i}$ according to the values in the technical program $X_{P i}$.

The angular reference values on each axis $\delta \theta$ ri, processed by the PLC multiprocessor system (SM-PLC), based on the mathematical model using the Denavit - Hartenberg method with determining the associated inverted Jacobean matrix, are sent in real time through the ARCNET communication network. The Cyber Physical Systems receives from the PC the reference trajectory position $\mathrm{X}_{\mathrm{Pi}}$ processed by the interpolator (ITP) into desired positions in the robot environment $X_{\mathrm{Di}}$ and the current angular values $\theta$ ci from the $\mathrm{PLC}_{0}$.

The incremental transducer interface (ITI) generates the actual position on each robot motion axis, using 6 specialized high speed counting modules with own central unit SA93, by the processing of signals from the incremental transducers (EN1-EN18) with $2,048 \mathrm{rot} / \mathrm{min}$ allowing 2,048,000 impulses per axis, for $0.01 \mathrm{~mm}$ resolution.

A wide variety of physical effects can be exploited in the construction of sensors. Recent years have seen the design of a huge range of sensors, and much of the progress in designing smart systems can be attributed to modern sensor technology. The VIPRO platform mainly uses the following analogical and digital sensors input signals:

- incremental position transducers, which measure the joint variables, respectively the relative positions of the kinematic elements adjacent to the conducting joints;

- inertial transducers and torque transducers, 3 for each of the two mechanisms of the modular leg, for dynamic control of the stability of the robot walking;

- force transducers $\left(\mathrm{FS}^{1}, \ldots, \mathrm{FS}^{6}\right)$, which measure with 12-bit resolution the reaction forces between the terrain and the legs, in their extremity points;

- piezo-ceramic transducers, with the aim of maintaining static equilibrium of the leg mechanisms;

- inertial vertical attitude transducers for maintaining robot stability and the platform's horizontal position.

The position controllers (CPki, i=1-3, $\mathrm{k}=1-6$ ) ensure motion control on each freedom axis based on the reference points $\mathrm{X}_{\mathrm{Di}}$ generated by the interpolator module (ITP) in the PCOAH software.

The actual position error is determined in real time in the PLC multiprocessor system (SMPLC) using the PLC1-PLC5 controllers through processing of the Jacobean matrix on each robot axis, based on direct kinematics through the Denavit - Hartenberg method and the associated inverted matrix.

The $\mathrm{PC}-\mathrm{OAH}$ (PC-OPEN Architecture) system by using a real time ExTR "multitask" (multiprogram) executive allows flexibility for the Cyber Physical Systems by integrating new functions the basic functions: interpolation (ITP), main program (PP) for user interface, compliant control programs through the fuzzy multi-stage method (CTRL C), prehensive control with tracking functions (CTRL P), working mode control for cooperation in 
obstacle avoidance (CWC), slope walking or obstacle avoidance control (WSC), optimal predictive control (OPC), respectively intelligent control interfaces ICNs, ICEx. A digital camera interface for image recognition and remote communication interfaces through radio modem data transmission, GSM or wireless systems can be configured.

The Cyber Physical Systems of the versatile, intelligent and portable robot platform VIPRO implemented through the ICF1-ICF7 control interfaces allow a stable movement of the robot, improving the dynamic for ensuring robot leg compliance in movement on uncertain and uneven terrain. Using these control interfaces allow for a decentralization of the control system, through the strategy for trajectory generation by a central command for walking mode control, followed by multiplexing the local control rules implemented in interface ICF1-ICF7.

\section{Results and Conclusion}

The Cyber Physical Systems integrated into the open architecture and intelligent interfaces of the VIPRO platform leads to improved performance in robot and nano/micro/macro manipulator control, ensuring a high level of versatility for the platform in transforming from the base function of real time movement, navigation and orientation control, to intelligent functions for 3D virtual robot control through a number of functions, optimized and tested on the platform system.

More over, the portability of the VIPRO platform allows the possibility of transfering the intelligent control interfaces of the 3D virtual robot and environment from the PC system of an external remote user to the VIPRO platform, as well as the remote control of the VIPRO platform by an external user, in the absence of the mechanical structure, ensuring the design, testing and experimentation of control methods on an existing classical mechatronic device and control system, without the need for modifying its hardware structure.

In continuing this trend the advent of ubiquitous embedded computing, sensing, and wireless networking technologies are becoming the key enabling technologies with regard to the interaction, real time control and construction of physical engineering systems applied to robotics. The main results and researches lead to the conclusion that the Cyber Physical Systems applied to the VIPRO platform bring greater results than other similar virtual platforms, but will also allow the design, testing and experimentation of intelligent control methods in real time, thereby providing the opportunity for the VIPRO platform to enter the IT market as a new component among existing platforms.

\section{Acknowledgements}

This work was accomplished through the Partnerships Program in priority fields - PN II, developed with the support of MENUEFISCDI，PN-II-PT-PCCA-2013-4，ID2009, VIPRO project no.009/2014, Romanian Academy and FP7-PEOPLE-2012-IRSES RABOT project no. 318902.

The work has been funded by the Sectoral Operational Programme Human Resources Development 2007-2013 of the Ministry of European Funds through the Financial Agreement POSDRU/159/1.5/S/132397.

\section{REFERENCES}

1. KAJITA, S., F. KANEHIRO, K. KANEKO, K. FUJIWARA, K. HARADA, K. YOKOI, H. HIRUKAWA, Biped Walking Pattern Generation by using Preview Control of Zero-Moment Point, Proceedings of the 2003 Intl. Conference on Robotics and Automation, Taipei, 2003, pp. 1620-1626.

2. KAGAMI, S., K. NISHIWAKI, J. KUFFNER, Y. KUNIYOSHI, M. INABA, H. INOUE, Online 3D Vision, Motion Planning and Bipedal Locomotion Control Coupling System of Humanoid Robot H7, Proceedings of the 2002 IEEE/ RSJ International Conference on Intelligent Robots and Systems, Lausanne, Switzerland, 2002, pp. 2557-2562.

3. VLADAREANU, L., G. TONT, I. ION, M. S. MUNTEANU, D. MITROI, Walking Robots Dynamic Control Systems on an Uneven Terrain, Advances in Electrical and Computer Engineering, vol. 10, no. 2, 2010, pp. 146-153. 
4. KIM, J. Y, I. W. PARK, J. H. OH, Experimental Realization of Dynamic Walking of the Biped Humanoid Robot KHR-2 using Zero Moment Point Feedback and Inertial Measurement, Advanced Robotics, Vol. 20, No. 6, 2006, pp. 707-736.

5. VLADAREANU, L., I. ION, L. M. VELEA, D. MITROI, A. I. GAL, The Real Time Control of Modular Walking Robot Stability, Recent Advances in Electrical Engineering, Proceedings of the $8^{\text {th }}$ International Conference on Applications of Electrical Engineering (AEE '09), Houston, USA, pg.179-186.

6. VLADAREANU, V., O. I. SANDRU, VLADAREANU, H. N. YU, Extension Dynamical Stability Control Strategy for the Walking Robots, International Journal of Technology Management, SKIMA 2013, Inderscience Publisher, pp. 1741-5276.

7. VLADAREANU, L., L. CAPITANU, Hybrid Force-Position Systems with Vibration Control for Improvement of Hip Implant Stability, Journal of Biomechanics, 45, S1, S279.

8. DUMITRACHE, I., S. I. CARAMIHAI, A. STANESCU, From Mass Production to Intelligent Cyber-Enterprise, Control Systems and Computer Science (CSCS), 2013 19th International Conference on, pp. 399-404, Print ISBN: 978-1-4673-6140-8.

9. MOISESCU, M. A., I. S. SACALA, A. M. STANESCU, C. SERBANESCU, Towards Integration of Knowledge Extraction Form Process Interoperability in Future Internet Enterprise Systems, 2012/5/23, Information Control Problems in Manufacturing, vol. 14(1), pp. 1458-1463.

10. SACALA, I. S., M. A. MOISESCU, D. REPTA, Towards the Development of the Future Internet Based Enterprise in the Context of Cyber-Physical Systems, Control Systems and Computer Science (CSCS), 2013 19th International Conference, pp. 405-412.

11. POP, N., L. VLADAREANU, I. N. POPESCU, C. GHIȚĂ, I. A. GAL, S. CANG, H. N. YU, V. BRATU, M. DENG, A Numerical Dynamic Behaviour Model for 3D Contact Problems with Friction,
Computational Materials Science, vol. 94, November 2014, pp. 285-291.

12. Duan, J. C., F. L. Chung, A Mamdani Type Multistage Fuzzy Neural Network Model, IEEE Fuzzy Systems Conference, FUZZ '98, 1998, pp. 1253-8.

13. VLADAREANU, V., G. TONT, L. VLADAREANU, F. SMARANDACHE, The Navigation of Mobile Robots în Non-Stationary and Non-Structured Environments, International Journal of Advance Mechatronic Systems vol. 5(4), 01/2013, pp. 232-243.

14. VLADAREANU, V., P. SCHIOPU, S. CANG, H. N. YU, Reduced Base Fuzzy Logic Controller for Robot Actuators, Applied Mechanics and Materials, Trans Tech Publications, Switzerland, vol. 555, 2014, pp. 249-258.

15. SANDRU, V., C. G. CONSTANTINESCU, M. BOSCOIANU, The use of Analytic Hierarchy Process for the life extension analysis of Air Defense Integrated Systems, Recent Advances in Applied Mathematics, Modelling and Simulation, pp. 203-211, ISBN: 978-960-474-398-8.

16. VLADAREANU, V., O. I. SANDRU, P. SCHIOPU, A. SANDRU, L. VLADAREANU, Extension Hybrid Force-Position Control of Mechatronics Systems, First International Symposium of Extenics, Beijing 2013.

17. XIAOJIE, W., W. XIAOYUN, H. N. YU, W. HONGBO, L. LING, L. VLADAREANU, O. MELINTE, Kinematics Analysis for the Leg Mechanism of a Wheel-leg Hybrid Rescue Robot, 2014 UKACC International Conference on Control (CONTROL 2014), Loughborough, U.K., 9-11 July 2014, pp. 369-373, IEEE, ISBN 978-1-4799-2518-6.

18. VLADAREANU. L., L. M. VELEA, R. I. MUNTEANU, A. CURAJ, S. CONONOVICI, T. SIRETEANU, L. CAPITANU, M. S. MUNTEANU, Real Time Control Method and Device for Robots in Virtual Projection, Patent no. EPO-09464001, 18.05.2009, EP2105263. Patent OSIM 123527/30.04.2013 
19. CAI, W., Extension Set and NonCompatible Problems, Journal of Scientific Exploration, 1983(1), pp. 83-97.

20. CHUNYAN, Y., W. CAI, Extension Engineering, Beijing: Science Press, 2007, p. 357.

21. SMARANDACHE, F., Extenics in Higher Dimensions, Institute of Extenics and Innovation Methods, Guangdong University of Technology, ISBN: 9781599732039

22. VLADAREANU, V., P. ȘCHIOPU, L. VLADAREANU, Theory and Application of Extension Hybrid ForcePosition Control in Robotics, U.P.B. Sci. Bull., Series A, vol. 76(3), 2014, pg.43-54, ISSN 1223-7027.

23. SMARANDACHE, VLADAREANU,

F., Applications of Neutrosophic Logic to Robotics - An Introduction, The 2011 IEEE International Conference on Granular Computing Kaohsiung, Taiwan, Nov. 8-10, 2011, pp. 607-612, ISBN 978-1-4577-0370-6
24. VLADAREANU, L., The Robots' Real Time Control through Open Architecture Systems, Topics in Applied Mechanics, vol. 3, cap.11, Published by Ed. Academiei 2006, pp. 460-497.

25. GILBERT, S., N. LYNCH, S. MITRA, T. NOLTE, BSelf-stabilizing Robot Formations over Unreliable Networks, ACM Trans. on Autonomous and Adaptive Systems (TAAS) TAAS Homepage archive, Volume 4 Issue 3, July 2009.

26. MOISESCU, M. A., I. S. SACALA, Towards the Development of Interoperable Sensing Systems for the Future Enterprise. Journal of Intelligent Manufacturing, 2014, pp. 1-22.

27. THORSTEINSSON, G., T. PAGE, A. NICULESCU, Using Virtual Reality for Developing Design Communication, Studies in Informatics and Control, vol. 19, no. 1, 2010, pp. 93-106. 